\title{
Avaliação do impacto da dor crônica na qualidade de vida dos pacientes antes e após hernioplastia inguinal
}

\author{
Evaluation of the impact of chronic pain on patients' quality of life \\ before and after inguinal hernioplasty
}

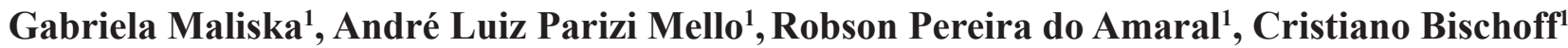

Maliska G, Mello ALP, Amaral RP, Bischff C. Avaliação do impacto da dor crônica na qualidade de vida dos pacientes antes e após hernioplastia inguinal / Evaluation of the impact of chronic pain on patients' quality of life before and after inguinal hernioplasty. Rev Med (São Paulo). 2019 jan.-fev.;98(1):40-5.

RESUMO: Este trabalho tem como objetivo avaliar o impacto da dor crônica na qualidade de vida dos pacientes antes e após serem submetidos à hernioplastia inguinal (HPI). Apresenta-se como uma coorte prospectiva, composta por 67 pacientes submetidos à hernioplastia inguinal por meio da técnica de Lichtenstein. Foram avaliados pelo questionário de qualidade de vida SF-36, Escala de Estimativa Numérica da Dor (EEND) e instrumento de coleta de dados. A coleta ocorreu em duas etapas, a primeira antes da cirurgia de HPI e a segunda 3 meses após. Dados qualitativos foram apresentados na forma de frequência simples e relativa, quantitativos em média e desvio padrão. Como resultados obtidos, a prevalência total da dor reduziu de $95,52 \%$ para $53,73 \%$ após três meses da HPI. $41,79 \%$ permaneceram com dor leve após HPI, $10,45 \%$ moderada, $1,48 \%$ intensa e nenhum paciente dor insuportável. $80,60 \%$ dos pacientes que apresentavam dor obtiveram o êxito de reduzir o nível após HPI. Dentre os domínios do SF36, aqueles que apresentaram significância alteraram a média da seguinte maneira: aspecto físico, dor, capacidade funcional, saúde mental, aspectos sociais e aspectos emocionais. Os domínios: estado geral de saúde e vitalidade não obtiveram significância. A dor crônica não proporcionou grande impacto na qualidade de vida dos pacientes após a HPI. A redução na prevalência e intensidade da dor após a cirurgia, a melhora ao desempenhar suas atividades e de sua imagem corporal são os fatores impulsores na grande melhora da qualidade de vida dos pacientes.

Descritores: Hérnia inguinal/cirurgia; Dor crônica/cirurgia; Medição da dor; Qualidade de vida.
ABSTRACT: This study aims at evaluating the impact of chronic pain on patient's quality of life before and after undergoing inguinal hernioplasty (HPI). It is presented as a prospective cohort, composed of 67 patients submitted to inguinal hernioplasty, through Liechtenstein technique. The patients were evaluated using the SF-36 quality of life questionnaire, numerical pain rating scale (NPRS) and data collection tools. The collection occurred in two steps. The first step before the surgery and the second step 3 months later. Qualitative data was presented as simple and relative frequency, quantitative data was presented in average and standard deviation. As obtained outcomes, total pain prevalence reduced from $95.52 \%$ to $53.73 \%$ after three months of IHP. $41.79 \%$ remained with mild pain after HPI, $10.45 \%$ moderate, $1.48 \%$ severe and no patient reported unbearable pain. $80.60 \%$ of the patients who were in pain, succeeded in reducing the level after HPI. Among the SF36 domains, those who presented relevance, altered the average as follows: physical appearance, pain, functional capacity, mental health, social aspects and emotional aspects. The domains general health and vitality were not significant. Chronic pain did not have a great impact on patient's quality of life after IHP. The reduction in the prevalence and intensity of pain after surgery, improvement in performing their activities and their body image are the driving factors in the great improvement of patient's quality of life.

Keywords: Hernia, inguinal/surgery; Chronic pain/surgery; Pain measurement; Quality of life.

Apresentado no V Congresso Brasileiro de Hérnia, Foz do Iguaçu, PR, 21 abr. 2018.

Fonte de financiamento: Não há.

1. Hospital Regional Homero de Miranda Gomes. ORCID: Maliska G - https://orcid.org/0000-0002-8774-7144; Mello ALP - https:// orcid.org/0000-0002-0162-1461. Email: gabimaliska@hotmail.com; melloalp@gmail..com; dr_robsondoamaral@hotmail.com; cristianobischoff@gmail.com.

Endereço para correspondência: Gabriela Maliska Rua Antônio Costa, 11. Apto 305 B. Bairro Itacorubi. Florianópolis, SC. CEP: 88034-070. Email: gabimaliska@hotmail.com. 


\section{INTRODUÇÃO}

A hérnia de parede abdominal ocorre quando há deslocamento de conteúdo através de um orifício, o anel herniário, e a consequente invasão de um espaço indevido, o saco herniário. Esse deslocamento somente é possível em razão do enfraquecimento da musculatura abdominal, por problema congênito ou associado a esforços em demasia ${ }^{1}$. As hérnias mais frequentes são as inguinais (70-75\%), as femorais (6-17\%), as umbilicais (3-8,5\%), as incisionais $(10 \%)$, as epigástricas $(1-6 \%)^{2,3}$. No Brasil, segundo dados do Ministério da Previdência Social, a hérnia inguinal é responsável por até 80 mil afastamentos por ano, sendo a terceira doença que mais compromete os trabalhadores ${ }^{4}$.

O tratamento cirúrgico de hérnia inguinal é um dos procedimentos mais realizados mundialmente. Dos 20 milhões de reparos anuais, 1 milhão ocorre nos Estados Unidos $^{5}$. Os dados do Serviço Nacional de Saúde da Inglaterra, entre 2011 a 2012, relatam que foram realizadas 70 mil inguinais, 17,000 umbilicais, 8000 incisionais e 6000 outras reparações de hérnia ventral ${ }^{6}$.

A forma de tratamento das hérnias se dá por meio da hernioplastia, cabendo a Edoardo Bassini o mérito de precursor, em 18907. Em 1986, Lichtenstein ${ }^{8}$ criou uma técnica pioneira no reparo das hérnias inguinais, utilizando uma "prótese em rede" e desenvolvendo uma cirurgia livre de tensão. Com o advento de novas técnicas cirúrgicas e implante de telas, verificou-se uma significante redução na recidiva $^{9,10}$. Devido a esta redução, atualmente o enfoque dos estudos relacionados à hérnia inguinal voltou-se para a principal consequência pós-operatória, a dor crônica ${ }^{11}$.

Segundo a Associação Internacional para Estudos da Dor (IASP), dor é uma experiência sensorial e emocional desagradável associada a um dano real ou potencial dos tecidos, ou descrita em termos de tais lesões. A dor crônica é definida como a dor contínua ou recorrente de duração mínima de três meses, muitas vezes não desaparece com o emprego dos procedimentos terapêuticos convencionais e é causa de incapacidades e inabilidades prolongadas ${ }^{12}$.

A dor crônica após HPI está relacionada com a lesão tecidual e neuronal locais durante a cirurgia, assim como o processo cicatricial produzido pela sutura ou pela própria tela ${ }^{12-14}$. Esta complicação apresenta taxa de incidência que varia de $11 \%$ para $60,8 \%{ }^{15-17}$, impactando na qualidade de vida do paciente. Dentre os fatores de risco que desenvolvem dor crônica, destacam-se fatores psicossociais, idade jovem e o elevado nível de dor antes da cirurgia ${ }^{18-21}$.

A dor crônica incapacita as atividades laborais, acarretando problemas sociais e econômicos, bem como para a saúde pública ${ }^{22}$. O afastamento por doença tem um efeito negativo sobre a economia, devido à redução da força de trabalho e o aumento dos custos de produção, médico, previdenciário e social. O absenteísmo gerado está relacionado com alterações no campo físico, psicológico e social dos estudos da qualidade de vida da população de trabalhadores ${ }^{23}$.

A qualidade de vida tem se tornado um critério importante na avaliação da efetividade de intervenções e tratamentos na área de saúde, e no impacto das doenças crônicas no cotidiano das pessoas ${ }^{24-26}$. Para melhor analisar a qualidade de vida, é necessário explorar domínios como capacidade funcional, aspectos físicos, dor, estado geral da saúde, vitalidade, aspectos sociais, aspectos emocionais e saúde mental, que fazem parte do questionário genérico de avaliação de qualidade de vida SF-36 (Brasil SF-36) ${ }^{27}$.

Diante da alta incidência mundial desta doença e da possível interferência na qualidade de vida destes indivíduos provocando, assim, problemas sociais e econômicos, este trabalho visa avaliar o impacto da dor crônica na qualidade de vida dos pacientes antes e após serem submetidos à hernioplastia inguinal.

\section{MÉTODO}

Este trabalho trata-se de um estudo observacional, do tipo coorte prospectiva, realizado no ambulatório de cirurgia do Hospital Regional Homero de Miranda Gomes, localizado na cidade de São José - SC.

A amostra foi composta de 67 pacientes de ambos os sexos, todos com diagnóstico de hérnia inguinal e submetidos à cirurgia de HPI através da técnica de Lichtenstein $^{8}$, no período de dezembro de 2016 a julho de 2017. Foram excluídos da amostra indivíduos incapazes de responder ao questionário (por déficit auditivo ou cognitivo) ou que apresentaram hérnia recidivada. A seleção dos participantes da amostra foi realizada de forma consecutiva.

$\mathrm{Na}$ primeira etapa, antes da cirurgia de HPI, foi aplicado o questionário genérico de avaliação de qualidade de vida SF-36 (SF-36) ${ }^{27}$, a Escala de Estimativa Numérica da Dor (EEND) ${ }^{28}$ e o instrumento de coleta de dados, elaborado especificamente pelos pesquisadores para este estudo. Neste instrumento foram coletadas informações relacionadas ao sexo, idade e comorbidades prévias.

$\mathrm{Na}$ segunda etapa, três meses após a HPI, foi aplicado novamente o questionário SF-36 e a EEND, bem como coletadas as informações em prontuário médico sobre tempo de repouso e possíveis complicações ocorridas após a cirurgia.

O questionário SF36 foi respondido pelos pacientes auxiliados por profissional devidamente treinado. Foi avaliado a pontuação que o participante obteve em cada questão e efetuado o cálculo do Raw Scale, onde o valor das questões dos 8 domínios variaram de 0 (zero) a 100 (cem), onde 0 é a pior e 100 a melhor pontuação para cada domínio ${ }^{27}$.

A EEND permitiu ao paciente expressar numericamente a intensidade da dor que apresentou; 
recomenda-se o uso da escala numérica em pesquisas envolvem pacientes com dor crônica ${ }^{28}$.

A tabulação e tratamento primário dos dados foi realizada no programa Windows Excel e posteriormente analisados pelo programa Software Statistical Package for the Social Sciences (SPSS) Version 18.0. [Computer program]. Chicago: SPSS Inc; 2009.

Os dados qualitativos foram apresentados na forma de frequência simples e relativa e os quantitativos, em média e desvio padrão. Foi realizada a associação da variável de exposição com a incidência dos desfechos de interesse, os domínios da SF-36. Para comparação entre médias, entre o primeiro e segundo tempo, foi utilizado o teste $t$ de student, com nível de significância de $\mathrm{p} \leq 0,05$.

$\mathrm{O}$ estudo foi aprovado pelo Comitê de Ética em Pesquisa da Universidade do Sul do Estado (UNISUL), sob o registro CAAE 62460716.9.0000.5369. Os pesquisadores declaram ausência de conflitos de interesse.

\section{RESULTADOS}

A amostra obtida foi composta por 67 pacientes, selecionados a partir de 79 indivíduos, dos quais 12 deixaram de participar por não comparecerem à segunda entrevista. Todas as cirurgias foram realizadas pela mesma equipe cirúrgica e quanto à coleta de dados, foram realizadas pelo mesmo entrevistador.

Na caracterização da amostra, houve predomínio de pacientes masculinos $(94,0 \%)$, dos quais $55,2 \%$ estão na faixa etária está entre 40 e 60 anos, sendo $28,4 \%$, menor que 40 anos e 16,4\%, com mais de 60 anos.

Dentre as comorbidades, foram identificadas nos pacientes: hipertensão arterial sistêmica (20,9\%), dislipidemia $(8,9 \%)$, depressão $(7,5 \%)$ e diabetes mellitus $(6,0 \%)$.

Dentre complicações pós-operatórias, 4,5\% dos pacientes apresentaram infecção da ferida operatória; $4,5 \%$, seroma; e 3\% deiscência de pontos. Quanto ao retorno às atividades, 68,6\% dos pacientes voltaram em mais de 60 dias, $28,4 \%$ entre 30 e 60 dias, e $3 \%$ em menos de 30 dias.

Segundo a escala da dor: No pré-operatório, houve predomínio de pacientes com dor moderada; seguido de dor fraca, dor intensa e dor insuportável. Após três meses da cirurgia, houve predomínio de pacientes sem dor; pacientes com dor fraca e moderada, representaram juntos aproximadamente metade dos pacientes. Vale ressaltar que três meses após a cirurgia apenas 1,49\% apresentaram dor intensa e nenhum paciente dor insuportável (Tabela 1). A prevalência de pacientes com algum tipo de dor antes da cirurgia foi de $95,52 \%$, reduzindo para $53,73 \%$ após o procedimento.

Tabela 1. Intensidade de dor avaliada pela Escala de Estimativa Numérica da Dor antes e três meses após hernioplastia inguinal em pacientes atendidos em São José - SC, no período de dezembro de 2016 a julho de 2017

\begin{tabular}{lcccc}
\hline Intensidade da dor & Antes & $\mathbf{\%}$ & $\mathbf{3}$ meses & $\mathbf{\%}$ \\
\hline Sem dor & 3 & 4,48 & 31 & 46,27 \\
Dor fraca & 16 & 23,88 & 28 & 41,79 \\
Dor moderada & 33 & 49,25 & 7 & 10,45 \\
Dor intensa & 14 & 20,89 & 1 & 1,49 \\
Dor insuportável & 1 & 1,49 & - & - \\
Total & 6 & 100 & 67 & 100 \\
\hline
\end{tabular}

Os resultados obtidos na alteração das variáveis da EEND pós-hernioplastia mostram maior representatividade de pacientes que reduziram um nível na classificação da dor, seguida pela redução em dois níveis. Os pacientes que mantiveram a mesma classificação da dor foram $14,92 \%$; enquanto que apenas 4,48\% aumentaram um nível (Tabela 2).

Dentre os domínios do SF36, comparando a qualidade de vida antes e após três meses da cirurgia, destacam-se o aspecto físico, que variou de 25,37 para 84,33 , e o domínio dor, que aproximadamente dobrou. Os domínios capacidade funcional, saúde mental, os aspectos sociais e emocionais, obtiveram significância estatística. Os domínios: estado geral de saúde e vitalidade não obtiveram significância estatística (Tabela 3).
Tabela 2. Frequência de alteração na Escala de Estimativa Numérica da Dor após HPI em pacientes atendidos em São José - SC, no período de dezembro de 2016 a julho de 2017

\begin{tabular}{lcc}
\hline $\begin{array}{l}\text { Frequência de alteração na } \\
\text { escala da dor }\end{array}$ & N & \% \\
\hline Aumento de 1 nível & 3 & 4,48 \\
Redução de 1 nível & 30 & 44,78 \\
Redução de $\mathbf{2}$ níveis & 16 & 23,88 \\
Redução de 3 níveis & 8 & 11,94 \\
Manteve mesmo nível & 10 & 14,92 \\
Total & 67 & 100 \\
\hline
\end{tabular}


Maliska G, et al. Avaliação do impacto da dor crônica na qualidade de vida dos pacientes.

Tabela 3. Média e desvio padrão dos domínios do questionário SF-36, antes e após a cirurgia de hernioplastia inguinal em pacientes atendidos em São José - SC, no período de dezembro de 2016 a julho de 2017

\begin{tabular}{lccc}
\hline Domínios do questionário SF36 & $\begin{array}{c}\text { Antes da HPI } \\
\text { MÉDIA ( } \pm \text { DP) }\end{array}$ & $\begin{array}{c}\text { meses após HPI } \\
\text { MÉDIA ( } \pm \text { DP) }\end{array}$ & p* \\
\hline Capacidade funcional & $75,75(24,28)$ & $94,25(15,91)$ & $<0,001$ \\
Aspectos físicos & $25,37(37,31)$ & $84,33(33,38)$ & $<0,001$ \\
Dor & $41,48(20,56)$ & $83,81(19,43)$ & $<0,001$ \\
Estado geral de saúde & $72,70(18,44)$ & $75,60(21,09)$ & 0,055 \\
Vitalidade & $68,30(21,51)$ & $75,37(22,52)$ & 0,001 \\
Aspectos sociais & $73,47(24,61)$ & $86,42(27,57)$ & 0,015 \\
Aspectos emocionais & $56,96(45,41)$ & $93,53(119,23)$ & $<0,001$ \\
Saúde mental & $67,22(19,44)$ & $71,34(23,58)$ & \\
\hline
\end{tabular}

* Teste T de student, considerando significativo $\mathrm{p}<=0,05$

\section{DISCUSSÃO}

Os trabalhos de Fränneby ${ }^{18}$, Dennis ${ }^{19}$ e Powell ${ }^{29}$ apontam como preditivos de dor crônica os fatores psicossociais, desenvolvimento precoce de dor no pósoperatório, dor de grande intensidade antes da cirurgia, idade jovem, manipulação intraoperatória ou dano iatrogênico de nervos periféricos e a técnica cirúrgica por laparotomia aberta. No presente estudo, todos os pacientes que apresentavam depressão pré-operatória, metade dos pacientes que tinham dor intensa antes da HPI, e a maioria, $57,9 \%$ dos pacientes, com menos de 40 anos, evoluíram com dor crônica após HPI. Ao analisar a população, percebe-se que faixa etária jovem e pacientes com dor intensa estão presentes em número significativo na amostra, o que pode ter contribuído para aumento da probabilidade de a amostra desenvolver dor crônica.

Estudo prospectivo de Christoffersen et al. ${ }^{21}$ permite comparação com o presente estudo já que utiliza instrumento de coleta de dados e tempo de análise semelhantes; sendo um dos primeiros estudos a demonstrar mudanças significativas na qualidade de vida. O presente estudo demonstrou que a prevalência de pacientes com algum tipo de dor antes da cirurgia foi de $95,52 \%$, reduzindo para $53,73 \%$ após o procedimento. Destes pacientes que permaneceram com dor, $41,79 \%$ apresentavam dor fraca. A redução da dor repercutiu na significativa melhora da qualidade de vida referente ao domínio "aspecto físico".

Em seu estudo prospectivo com 5.506 pacientes, Courtney et al..$^{30}$ avaliaram a dor crônica após HPI e seu impacto na qualidade de vida utilizando o SF-36, obtendo melhora nos domínios relacionados a aspectos físicos e sociais, a capacidade funcional e a saúde mental. A coorte de Poobalan et al. ${ }^{31}$, de 226 pacientes, seguiu a mesma metodologia e verificou melhora após HPI nos domínios do SF-36: aspectos social, saúde mental e dor. Através de sua revisão literária, Campanelli ${ }^{32}$ afirma que a redução na dor aumenta a qualidade de vida dos pacientes após HPI. Isto pode ser verificado no presente estudo referente aos domínios dor, capacidade funcional e aspecto físico que obtiveram significância pelo fato de que $80,60 \%$ da sua população obteve o êxito em reduzir a intensidade da dor e, dos que permaneceram com dor, em $41,79 \%$ era de intensidade leve. Van Ramshorst ${ }^{33}$ verificou em sua coorte progressiva que a imagem construída pelo paciente sobre o seu corpo após a HPI interfere nos domínios aspectos sociais e aspecto emocional. Neste estudo houve uma melhora significativa destes domínios, pois a cirurgia de HPI permite resultados estéticos satisfatórios, melhorando a imagem sobre seu corpo, além de permitir que o mesmo desempenhe atividades antes limitadas pela hérnia inguinal.

Concluindo, apesar da alta prevalência no pósoperatório, a dor crônica não proporcionou grande impacto na qualidade de vida dos pacientes após a HPI. A redução na prevalência e intensidade da dor após a cirurgia, a melhora ao desempenhar suas atividades e de sua imagem corporal são os fatores impulsores na grande melhora da qualidade de vida dos pacientes.

Como limitante do trabalho, os autores citam o tempo de análise dos pacientes, propondo estender de três para seis meses a segunda etapa da entrevista. O estudo apresenta como pontos de relevância o fato de as cirurgias terem sido realizadas pela mesma equipe de cirurgiões, por meio da mesma técnica cirúrgica, e o mesmo entrevistador obteve os dados nos dois tempos da coorte.

Participação dos autores: Maliska G. idealizadora do projeto, responsável pela redação do artigo, coleta e análise dos dados. Mello ALP - suporte na correção do artigo e coleta dos dados. Amaral RP - cirurgião responsável pelas cirurgias de hernioplastia inguinal e auxiliar na correção do artigo. Bischff C. suporte na coleta e análise dos dados. 


\section{REFERÊNCIAS}

1. Desarda, MP. Concepts that prevent inguinal hernia formation - revisited new concepts of inguinal hernia prevention. Ann Emerg Surg. 2017;2(1):1007. Available from: https://pdfs. semanticscholar.org/99a5/5ee9378080524fa02b7b8bb21e bfeffe65c4.pdf?_ga $=2.92974495 .368948927 .1555076307$ 240929181.1555076307.

2. Dabbas N, Adams K, Pearson K, Royle G. Frequency of abdominal wall hernias: is classical teaching out of date? JRSM Short Rep. 2011;2(1):5. https://doi.org/10.1258/ shorts.2010.010071.

3. Townsend C, Beauchamp D, Evers M, Mattox KL, Sabiston DC. Sabiston Textbook of surgery: the biological basis of modern surgical practice. Philadelphia, PA: WB Saunders; 2001. doi: 10.1007/s10350-008-9293-5.

4. DataPrev [http://dadosabertos.dataprev.gov.br/]. Benefícios concedidos pela Classificação Internacional de Doenças (CID-10) [citado 24 set. 2017]. Disponível em: http:// dadosabertos.dataprev.gov.br/opendata/Ativ08/formato $=\mathrm{csv}$.

5. Cavazzola LT, Rosen MJ. Laparoscopic versus open inguinal hernia repair. Surg Clin North Am. 2013;93:1269-79. https:// doi.org/10.1016/j.suc.2013.06.013

6. Dew C. The Health and Social Care Information Centre Hospital Episode Statistics for England. Inpatient statistics, 2015-16. HESOnline 2016. Available at: http://www.hscic. gov.uk/searchcatalogue?productid $=9161 \&$ topics $=1 \% 2 \mathrm{fHos}$ pital + care $\% 2$ fInpatient + admissions\&sort=Relevance\&size $=10 \&$ page $=1 \#$ top

7. Campanelli G, Bertocchi V, Cavalli M, Bombini G, Biondi A, Tentorio T, et al. Surgical treatment of chronic pain after inguinal hernia repair. Hernia. 2013;17(3):347-53. https:// doi.org/10.1007/978-3-319-21587-7 28

8. Lichtenstein IL, Shore JM. Exploding the myths of hernia repair. Am J Surg. 1976;132(3):307-15. https://doi. org/10.1016/0002-9610(76)90381-0.

9. Jonge PVH, Lloyd A, Horsfall L, Tan R, O’Dwyer PJ. The measurement of chronic pain and health-related quality of life following inguinal hernia repair: a review of the literature. Hernia. 2008;12(6):561-9. https://doi.org/10.1007/s10029008-0412-y.

10. Sevonius D, Montgomery A, Smedberg S, Sandblom G. Chronic groin pain, discomfort and physical disability after recurrent groin hernia repair: impact of anterior and posterior mesh repair. Hernia. 2015;20(1):43-53. https://doi. org/10.1007/s10029-015-1439-5.

11. Chung L, O'Dwyer P. Pain and its effects on physical activity and quality of life before operation in patients undergoing elective inguinal and ventral hernia repair. Am J Surg. 2014;208(3):406-11. https://doi.org/10.1016/j. amjsurg.2014.02.011.

12. Montes A, Roca G, Sabate S, et al. Genetic and clinical factors associated with chronic postsurgical pain after hernia repair, hysterectomy, and thoracotomy a two-year Multicenter Cohort Study. Anesthesiology. 2015;122(5):1123-41. doi: 10.1097/ALN.0000000000000611.

13. Nikkolo C, Lepner U. Chronic pain after open inguinal hernia repair. Postgrad Med. 2015;128(1):69-75. https://doi.org/10. 1080/00325481.2016.1121090.
14. Maciel GSB, Simões RL, Carmo, FPTD, Garcia JWR, Paulo DNS. Resultados da herniorrafia inguinal bilateral simultânea pela técnica de Lichtenstein. Rev Col Bras Cir. 2013;40(5):370-3. https://doi.org/10.1590/S010069912013000500004

15. Nienhuijs S, Staal E, Strobbe L, Rosman C, Groenewoud $\mathrm{H}$, Bleichrodt R. Chronic pain after mesh repair of inguinal hernia: a systematic review. Am J Surg. 2007;194(3):394-400. https://doi.org/10.1016/j.amjsurg.2007.02.012.

16. Sanders D, Waydia S. A systematic review of randomised control trials assessing mesh fixation in open inguinal hernia repair. Hernia. 2013;18(2):165-76. https://doi.org/10.1007/ s10029-013-1093-8.

17. Shyam, DC, Rapsang, AG. Inguinal hernias in patients of 50 years and above. Pattern and outcome. Rev Col Bras Cir. 2013;40(5):374-9. http://dx.doi.org/10.1590/S010069912013000500005 .

18. Fränneby U, Sandblom G, Nordin P, Nyrén O, Gunnarsson U. Risk factors for long-term pain after hernia surgery. Ann Surg. 2006;244:212-9. doi: 10.1097/01.sla.0000218081.53940.01.

19. Dennis R, O'riordan D. Risk factors for chronic pain after inguinal hernia repair. Ann R Coll Surg Engl. 2007;89:218-20. https://doi.org/10.1308/003588407X178991

20. Singh AN, Bansal VK, Misra MC, Kumar S, Rajeshwari S, Kumar A, et al. Testicular functions, chronic groin pain, and quality of life after laparoscopic and open mesh repair of inguinal hernia: a prospective randomized controlled trial. Surg Endosc. 2012;26(5):1304-17. https://doi.org/10.1007/ s00464-011-2029-y.

21. Christoffersen M, Rosenberg J, Jorgensen L, Bytzer P, Bisgaard T. Health-related quality of life scores changes significantly within the first three months after hernia mesh repair. World J Surg. 2013;38(7):1852-9. https://doi. org/10.1007/s00268-013-2411-0.

22. Niraj G, Rowbotham DJ. Persistent postoperative pain: where are we now? Br J Anaesth. 2011;107:25-9. https://doi. org/10.1093/bja/aer116.

23. Henschke N, Kamper SJ, Maher CG. The epidemiology and economic consequences of pain. Mayo Clin Proc. 2015;90(1):139-47. doi: 10.1016/j.mayocp.2014.09.010.

24. Britton D. Prospective study of pain, quality of life and economic impact of open inguinal hernia repair. Brit J Surg. 2013;100(11):1489. https://doi.org/10.1002/bjs.9233

25. Farquhar M. Elderly people's definitions of quality of life. Social Sci Med. 1995;41(10):1439-46. https://doi. org/10.1016/0277-9536(95)00117-P.

26. Oliveira P, Duarte A, Guimarães A, Fernandes A, Ferreira C, Amorim D, et al. Chronic pain after outpatient inguinal hernioplasty: retrospective cohort study. Acta Med Port. 2018;31(11):624-32. https://doi.org/10.20344/amp.9381.

27. Ciconelli RM. Tradução para o português e validação do questionário genérico de avaliação de qualidade de vida Medical Outcomes Study 36-Item Short-Form Health Survey (SF-36) [Tese]. São Paulo: Universidade Federal de São Paulo - UNIFESP; 1997. http://repositorio.unifesp.br/ handle/11600/15360. 
Maliska G, et al. Avaliação do impacto da dor crônica na qualidade de vida dos pacientes.

28. Streiner DL, Norman GR, Cairney J. Health measurement scales: a practical guide to their development and use. 5 th ed. Oxford: Oxford University Press; 2015. p.38-65. doi: $10.1093 / \mathrm{med} / 9780199685219.001 .0001$.

29. Powell R, Johnston M, Smith WC, King PM, Chambers WA, Krukowski Z, et al. Psychological risk factors for chronic post surgical pain after inguinal hernia repair surgery: a prospective cohort study. Eur J Pain. 2012;16(4):600-10. https://doi.org/10.1016/j.ejpain.2011.08.010.

30. Courtney CA, DuVy K, Serpell MG, et al. Outcome of patients with severe chronic pain following repair of groin hernia. Br J Surg. 2002;89(10):1310-4. https://doi. org/10.1046/j.1365-2168.2002.02206.x.

31. Poobalan AS, Bruce J, King PM, et al. Chronic pain and quality of life following open inguinal hernia repair. $\mathrm{Br} \mathrm{J}$
Surg. 2001;88(8):1122-6. https://doi.org/10.1046/j.00071323.2001.01828.x.

32. Campanelli G, Bruni PG, Morlacchi A, Cavalli M. Chronic pain after inguinal hernia repair. In: Campanelli G, editor. Inguinal hernia surgery. Updates in surgery. Milano: Springer; 2017. p.157-68. https://doi.org/10.1007/978-88470-3947-6 16.

33. van Ramshorst GH, Eker HH, Hop WC, Jeekel J, Lange JF. Impact of incisional hernia on health-related quality of life and body image: a prospective cohort study. The American Journal of Surgery. 2012;204(2):144-50. https:// doi.org/10.1016/j.amjsurg.2012.01.012.

Recebido: 27.08 .18

Aceito:02.01.19 